\title{
Water Diffusion in Polymer Composites Probed by Impedance Spectroscopy and Time-Resolved Chemical Imaging
}

Hansen, Daniel; Brewer, Jonathan R.; Eiler, Johannes; Komjani, Niloufarsadat Mirmahdi; Hansen, Kristoffer; Thormann, Esben

\section{Published in:}

ACS Applied Polymer Materials

Link to article, DOI:

10.1021/acsapm.9b01107

Publication date:

2020

Document Version

Publisher's PDF, also known as Version of record

Link back to DTU Orbit

Citation (APA):

Hansen, D., Brewer, J. R., Eiler, J., Komjani, N. M., Hansen, K., \& Thormann, E. (2020). Water Diffusion in Polymer Composites Probed by Impedance Spectroscopy and Time-Resolved Chemical Imaging. ACS Applied Polymer Materials, 2(2), 837-845. https://doi.org/10.1021/acsapm.9b01107

\section{General rights}

Copyright and moral rights for the publications made accessible in the public portal are retained by the authors and/or other copyright owners and it is a condition of accessing publications that users recognise and abide by the legal requirements associated with these rights.

- Users may download and print one copy of any publication from the public portal for the purpose of private study or research.

- You may not further distribute the material or use it for any profit-making activity or commercial gain

- You may freely distribute the URL identifying the publication in the public portal 


\title{
Water Diffusion in Polymer Composites Probed by Impedance Spectroscopy and Time-Resolved Chemical Imaging
}

\author{
Daniel Hansen, ${ }^{\dagger} \ddagger \odot$ Jonathan R. Brewer, ${ }^{\S}$ Johannes Eiler, ${ }^{\dagger, \ddagger}$ Niloufarsadat Mirmahdi Komjani, ${ }^{\dagger} \|$ \\ Kristoffer Hansen, ${ }^{\ddagger}$ and Esben Thormann ${ }^{*},+0$
}

${ }^{\dagger}$ Department of Chemistry, Technical University of Denmark, 2800 Kgs. Lyngby, Denmark

‡Coloplast A/S, 3050 Humlebæk, Denmark

${ }^{\S}$ Department of Biochemistry and Molecular Biology, University of Southern Denmark, 5230 Odense M, Denmark

"Riemann A/S, 3400 Hillerød, Denmark

\author{
Supporting Information
}

\begin{abstract}
The ability to probe water diffusion in polymer composites is of significant importance for materials characterization and performance evaluation. Here, we investigate water diffusion in polymer composites using coherent anti-Stokes Raman scattering microscopy and impedance spectroscopy. Coherent anti-Stokes Raman scattering microscopy provides real-time information about evolution in the polymer composite structure during water uptake, which supports a method for evaluation of impedance data obtained in a water penetration

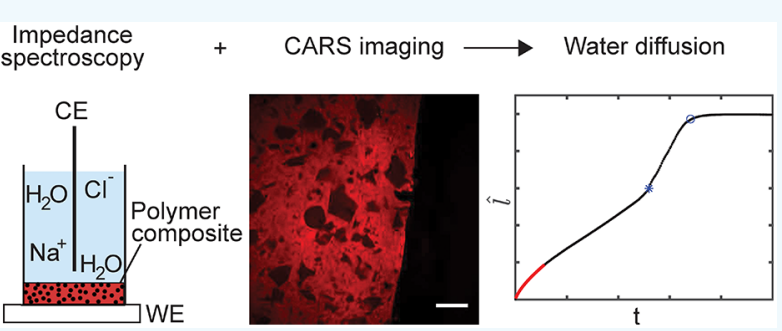
process. The method extracts the water diffusion kinetics from the relative change in the temporal capacitance, which includes contributions from changing dielectric properties and thickness. Specifically, the method is used for investigating two model composites relevant for medical adhesives consisting of a hydrophobic and rigid polymer matrix mixed with either poly(acrylic acid) or cetyl hydroxyethyl cellulose particles. Based on our analysis, these two composites exhibit anomalous diffusion and case II sorption, respectively, corresponding to faster relaxation processes in the poly(acrylic acid) containing composites compared to the cetyl hydroxyethyl cellulose containing composites during the water uptake process. Furthermore, the cetyl hydroxyethyl cellulose containing composites demonstrate characteristic penetration and saturation times almost an order of magnitude higher than the poly(acrylic acid) containing composites. In agreement with known chemical properties of these materials, this implies a lower water affinity for cetyl hydroxyethyl cellulose compared to poly(acrylic acid).
\end{abstract}

KEYWORDS: impedance spectroscopy, coherent anti-Stokes Raman scattering (CARS) microscopy, polymer composites, water diffusion, case II sorption, anomalous diffusion

\section{INTRODUCTION}

Polymer composites are used for a wide range of applications including paints, protective coatings, wind turbine blades, and adhesives. Such composites consist of a polymer matrix and fillers. One function of the matrix is to hold the geometrical shape of the composite, while the fillers give the material application-specific properties depending on the type of filler. Filler types include pigments for coloring, fibers for directional rigidity, and hydrophilic particles for enhanced water permeability. $^{1-5}$ Water diffusion in polymer composites is of great interest for manufacturing anticorrosive paints, protective coatings for microelectronics, hydrogels, and medical adhesives. $^{6-10}$ The ability to accurately probe water diffusion is required for rational material formulation and screening of material performance. Consequently, there exists a continuous drive to develop and refine experimental techniques for investigating water diffusion in polymer composites.

Diffusion studies are typically conducted using gravimetric measurements, while spectroscopic techniques represent an alternative approach. ${ }^{11-13}$ The choice of method depends on sample geometry, functionality, and time scale of diffusion. Obtaining accurate water uptake data presents a challenge using gravimetric measurements for degrading systems where significant sample mass is lost due to elution of species or cohesive fracture. ${ }^{14,15}$ Fredj et al. demonstrated the occurrence of leaching processes in marine coatings by comparing gravimetric measurements with impedance spectroscopy. Impedance spectroscopy proved not to be as sensitive to eluting species as gravimetric measurements and yielded more accurate uptake kinetics. ${ }^{16}$ Later, Grammatikos et al. emphasized the benefits of impedance spectroscopy to detect moisture uptake in fiber reinforced polymer composites without the contribution of sample loss. ${ }^{17}$ Other advantages of impedance spectroscopy include high time resolution, noninvasive nature, and the ability to detect electrochemical reactions. However, assumptions about the electrical behavior

Received: November 19, 2019

Accepted: December 23, 2019

Published: December 24, 2019 
of the system are required for physical interpretation. Complementary techniques should therefore be used in combination with impedance spectroscopy to ensure correct data interpretation. Reuvers et al. studied the water uptake in nylon films using electrochemical impedance spectroscopy and magnetic resonance imaging. The imaging was used to track the bulk water front since it was found to only detect moisture levels higher than 2\%. Meanwhile, impedance spectroscopy was found to be sensitive enough to detect low concentrations of water ahead of the bulk water front, which also made it suitable for studying the water penetration in the films. ${ }^{11}$

Experimentally obtained sorption curves are typically interpreted by using diffusion models to obtain quantitative information, such as the diffusion coefficient, which is useful for intersample comparison. Diffusion models can also help link together the material composition and performance, since they hold molecular information about the system. ${ }^{18}$ The nature of sorption kinetics strongly depends on the samplepenetrant interactions. ${ }^{19}$ Polymer systems with a high degree of chain mobility are typically above their glass transition temperature and can accommodate diffusion of penetrants with a high activity without the buildup of internal stress. Such systems will exhibit Fickian diffusion behavior. ${ }^{20}$ On the other hand, for polymeric systems where the time scales of the polymer relaxation processes are comparable with the time scales for penetrant diffusion, non-Fickian sorption kinetics are expected. These sorption processes will typically exhibit a sharp flow front propagating in the material. ${ }^{21-24}$

In the work presented here, we investigate polymer composites relevant for medical-grade adhesives composed of a hydrophobic matrix and hydrophilic particles. The water transport is expected to occur in the hydrophilic particles due to the low water permeability of the matrix. In this study, we select two different fillers that are expected to exhibit different water transporting properties due to differences in their hydrophilicity. Poly(acrylic acid) (PAA) was selected as a highly hydrophilic filler. PAA is typically categorized as a superabsorbent polymer, which is attributed to sodium ion dissociation upon wetting and its hydrophilic acid groups. Cetyl hydroxyethyl cellulose (CHEC) was chosen as a moderately hydrophilic particle due to its hydrophobic cetyl side chains and nonionic nature. ${ }^{25,26}$ We seek to understand how water interacts with the fillers by probing the diffusion kinetics using impedance spectroscopy and by imaging structural changes using coherent anti-Stokes Raman scattering (CARS) microscopy. CARS microscopy provides label-free chemical contrast by imaging specific molecular vibrations associated with particular chemical species. ${ }^{27}$ The technique is typically used for imaging dynamic processes in biological systems, since besides the chemical contrast, it also intrinsically yields high $3 \mathrm{D}$ spatial resolution and video-rate time resolution. ${ }^{28-30}$ We take advantage of CARS microscopy to obtain chemical contrast between fillers and matrix and probe real-time structural changes in the composite during water absorption. This allows us to probe the bulk water absorption indirectly by following the swelling of the hydrophilic particles, which will aid in the physical interpretation of the obtained impedance results. $^{31}$

\section{MATERIALS AND METHODS}

2.1. Materials. Polyisobutylene (PIB) was obtained from BASF (Germany), styrene-isoprene-styrene (SIS) was obtained from Kraton (Houston, TX), the poly(acrylic acid sodium salt) (PAA) particles were obtained from Steweart Superabsorbents (Hickory, $\mathrm{NC}$ ), and the cetyl hydroxyethyl cellulose (CHEC) particles were obtained from Ashland (Lexington, KY). The volume-weighted average diameter of each particle type was determined by laser diffraction (Malvern Mastersizer 2000, United Kingdom) to $d_{\mathrm{PAA}}=$ $68.4 \mu \mathrm{m}$ and $d_{\mathrm{CHEC}}=93.1 \mu \mathrm{m}$. Details on the laser diffraction measurements and the particle size distributions are provided in the Supporting Information (see Figure S1). PIB and SIS were mixed for $45 \mathrm{~min}$ at $30 \mathrm{rpm}$ and $90{ }^{\circ} \mathrm{C}$ under vacuum in a Brabender mixer (Brabender GmbH \& Co KG, Germany). The ratio between PIB and SIS was kept at 4:1 by weight, and the total mass of each mix was $60 \mathrm{~g}$. Particles were then mixed with the matrix to yield composites containing $40.0 \mathrm{wt} \%$ PAA particles corresponding to $27.1 \mathrm{vol} \%$. CHEC containing composites with the same volume fraction corresponding to 35.8 wt \% were also mixed. Particle volume fractions were calculated from particle densities $\rho_{\mathrm{PAA}}=1.6491 \pm$ $0.0003 \mathrm{~g} \mathrm{~cm}^{-3}$ and $\rho_{\mathrm{CHEC}}=1.3792 \pm 0.0003 \mathrm{~g} \mathrm{~cm}^{-3}$ obtained with an AccuPyc $1340 \mathrm{He}$ pycnometer from Micrometrics (Norcross, GA). After the particles were added to the matrix, vacuum was applied to minimize air entrapment in the composites. The mix was subsequently processed for $45 \mathrm{~min}$ at $30 \mathrm{rpm}$ and $90{ }^{\circ} \mathrm{C}$. After mixing, $20 \mathrm{~g}$ of material was pressed between two horizontal steel plates for $30 \mathrm{~s}$ under a load of 10 tons at $90{ }^{\circ} \mathrm{C}$. The sample mixes were pressed to thicknesses of $1050 \pm 25$ and $550 \pm 25 \mu \mathrm{m}$. The thickness was subsequently measured by using a thickness gauge (Mitutoyo Europe GmbH, Germany). After pressing, the desired sample geometry was obtained by punching. A saline solution for the impedance measurements was prepared from ultrapure water (arium pro, Satorius, Germany) and sodium chloride (Sigma-Aldrich, Denmark).

2.2. Impedance Spectroscopy. Impedance spectroscopy was performed by using a two-electrode system connected to an Autolab potentiostat (PGSTAT128N) with a frequency response analyzer module (FRA32M) both obtained from Metrohm Nordic (Denmark). The sample was adhered directly to a type $316 \mathrm{~L}$ stainless-steel plate (working electrode) and clamped to a glass cell (Figure 1a). A

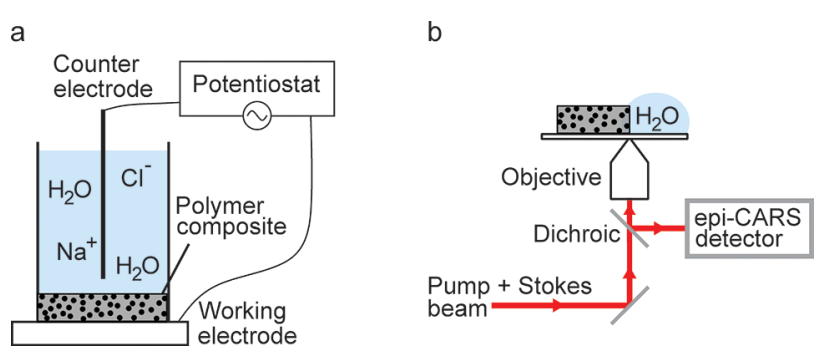

Figure 1. (a) Experimental setup for the impedance measurements consisting of a glass cell clamped to the polymer composite and working electrode. The counter electrode is fixed above the composite, which is immersed in a $154 \mathrm{mM} \mathrm{NaCl}$ solution. (b) Schematic illustration of CARS imaging of a polymer composite exposed to water. The CARS imaging is obtained through a pump and a Stokes beam, and the CARS signal is detected through the epiCARS detector.

graphite rod was used as both the counter and the reference electrode. The sample was exposed to $154 \mathrm{mM} \mathrm{NaCl}$ solution. The sample area was chosen to be $9.6 \mathrm{~cm}^{2}$, which is much larger than the particle sizes, to average out sample inhomogeneities. An oscillating potential with an amplitude of $10 \mathrm{mV}$ was applied between the two electrodes, and frequencies from $10 \mathrm{mHz}$ to $1 \mathrm{MHz}$ with one frequency per decade were scanned. A small potential was chosen to minimize perturbations of the water diffusion process. The measurements were done at room temperature, which was $23 \pm 2{ }^{\circ} \mathrm{C}$. We note that impedance results obtained after the electrolyte solution has penetrated through the entire polymer composite and reached the working electrode may have a significant contribution from the resistive and capacitive properties of an oxide layer at the surface of the working electrode. 

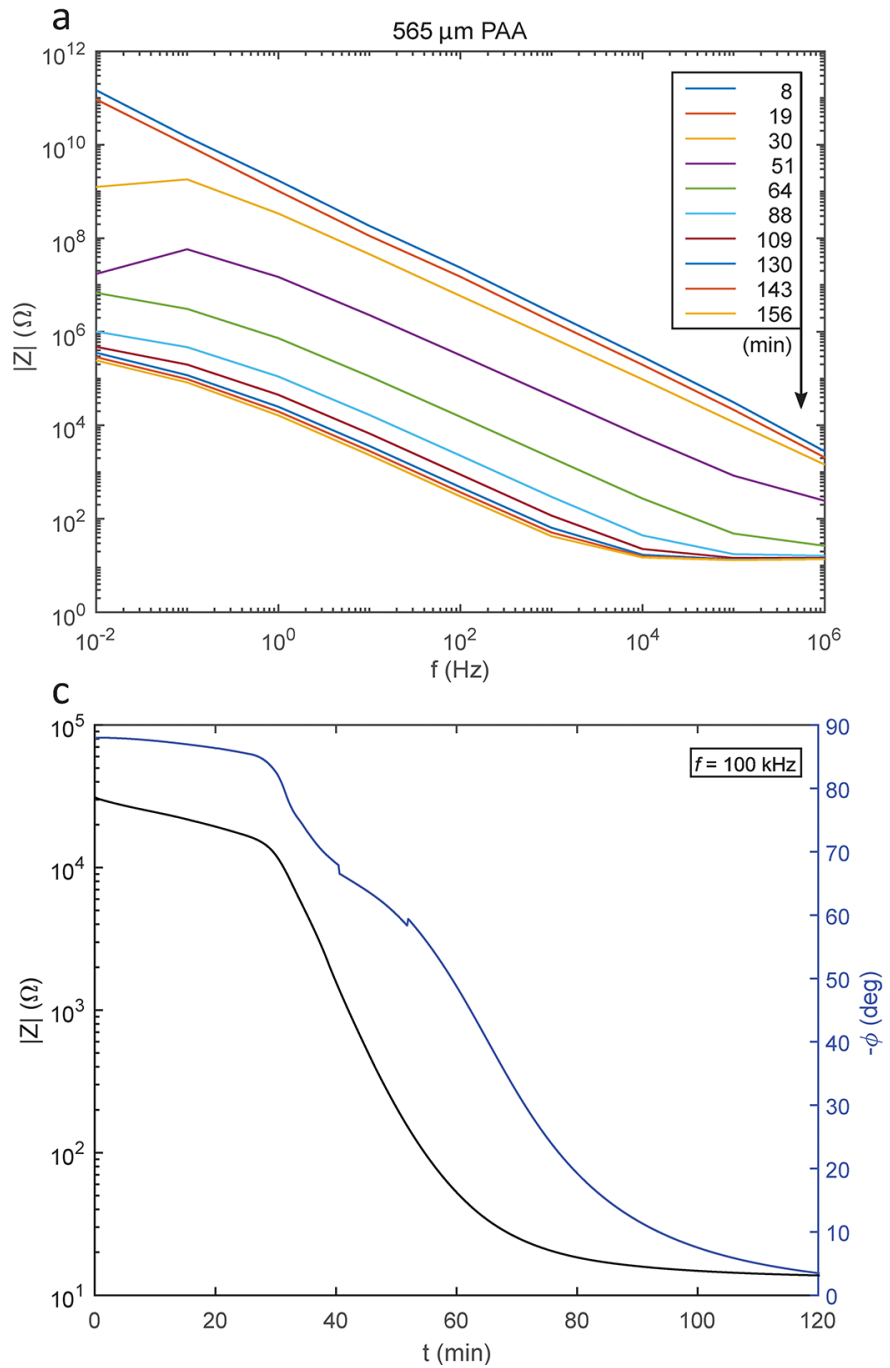

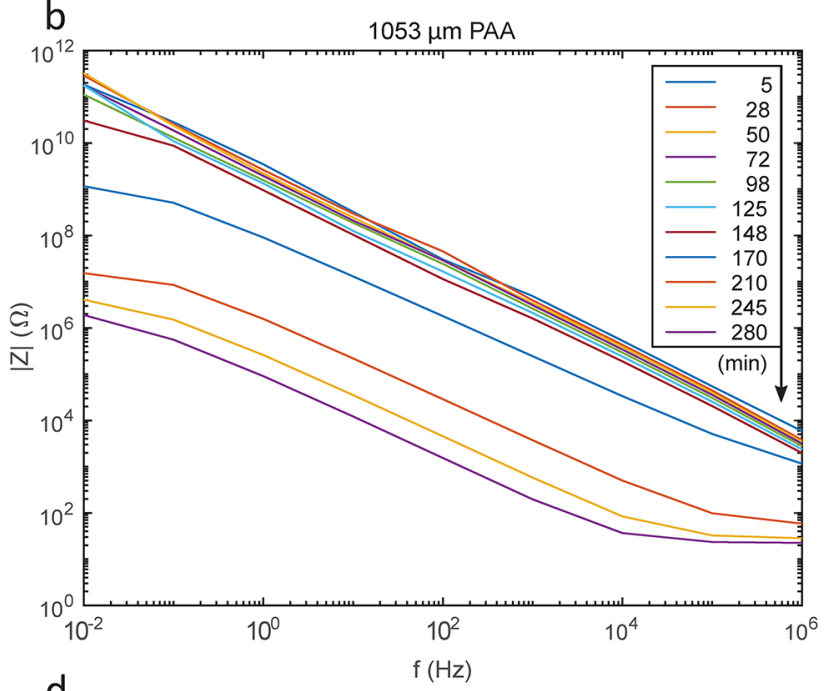

d

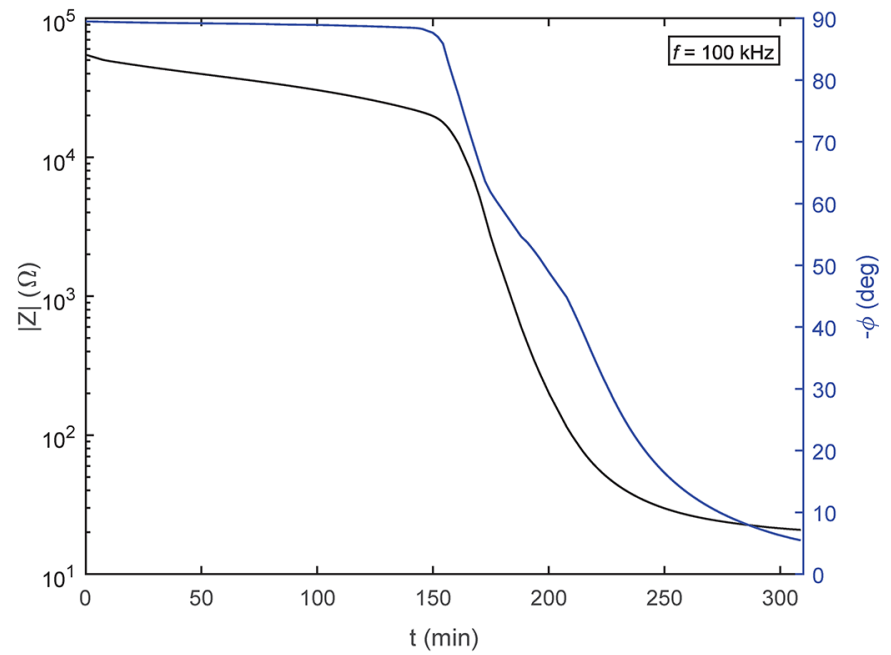

Figure 2. Bode (a, b) and impedance-time (c, d) plots of 565 and $1053 \mu \mathrm{m}$ PAA containing composites, respectively. The Bode plots are plotted at different times representing the entire duration of the experiments, while the impedance-time plots continuously show the impedance and phase angle as a function time at fixed frequency, $f=100 \mathrm{kHz}$.

However, the detailed diffusion analysis is performed for times much shorter than the time of penetration, and this contribution can therefore be considered insignificant since a thick layer (at least 250 $\mu \mathrm{m}$ ) of polymer composite remains on the working electrode and will dominate the impedance signal over the oxide layer.

The capacitance was obtained from the impedance by using an equivalent circuit model. Here, we model the polymer composite as a (RC) circuit, i.e., a resistor and a capacitor in parallel. The resistor and capacitor represent bulk conductivity and the dielectric properties of the sample, respectively. The resistance of the hardware and electrolyte solution is neglected. Additionally, the resistive and capacitive properties of the oxide at the working electrode surface can be neglected until the electrolyte solution is close to the working electrode. The capacitance, $C$, of a (RC) circuit is readily obtained as

$$
C=-\frac{Z^{\prime \prime}}{\omega\left(Z^{\prime 2}+Z^{\prime \prime 2}\right)}
$$

where $\omega$ is the angular frequency of the potential, $Z^{\prime}$ is the real part of the impedance, and $Z^{\prime \prime}$ is the imaginary part of the impedance.

2.3. CARS Imaging. CARS imaging was performed on a Leica TCS SP8 CARS microscope (Germany). A thick sample $(25 \times 25 \times 1$ $\mathrm{mm}^{3}$ ) was adhered to a coverslip and placed on the sample stage (Figure $1 \mathrm{~b}$ ). For the water absorption experiments, $50 \mu \mathrm{L}$ of water was placed at the edge of the sample, and the water was absorbed into the sample. All images were acquired with a field of view of $718 \times 718$ $\mu \mathrm{m}^{2}$ at room temperature $\left(23 \pm 2{ }^{\circ} \mathrm{C}\right)$. The highest chemical contrast was determined with a fixed Stokes laser at $1064 \mathrm{~nm}$, while varying the pump laser (PicoEmerald pump laser, APE, Germany). The pump laser was varied from 780 to $820 \mathrm{~nm}$ with $1 \mathrm{~nm}$ increments, resulting in wavenumbers ranging from 3400 to $2800 \mathrm{~cm}^{-1}$. The sample was exposed to water to investigate changes during water sorption. Here, image sequences were acquired at wavenumber $2997 \mathrm{~cm}^{-1}$ (pump laser: $807 \mathrm{~nm}$ ) with time intervals of $13.2 \mathrm{~s}$ and pixel size of $1.405 \times$ $1.405 \mu \mathrm{m}^{2}$. Image segmentation was done in MATLAB (The Mathworks, Inc.) by using the Image Processing toolbox. The images were binarized by using the im $2 b w$ function where the optimum threshold was determined from gray scale histograms, which were obtained using the imhist function.

\section{RESULTS AND DISCUSSION}

The goal of the present work is to develop a new method for probing water diffusion in polymer composites. This is done by first determining the temporal impedance of composites exposed to saline solution. Then, structural changes during the diffusion process are imaged by using CARS microscopy. From these findings, we finally describe a new model for impedance interpretation, which we use for comparing composites containing PAA and CHEC particles. 


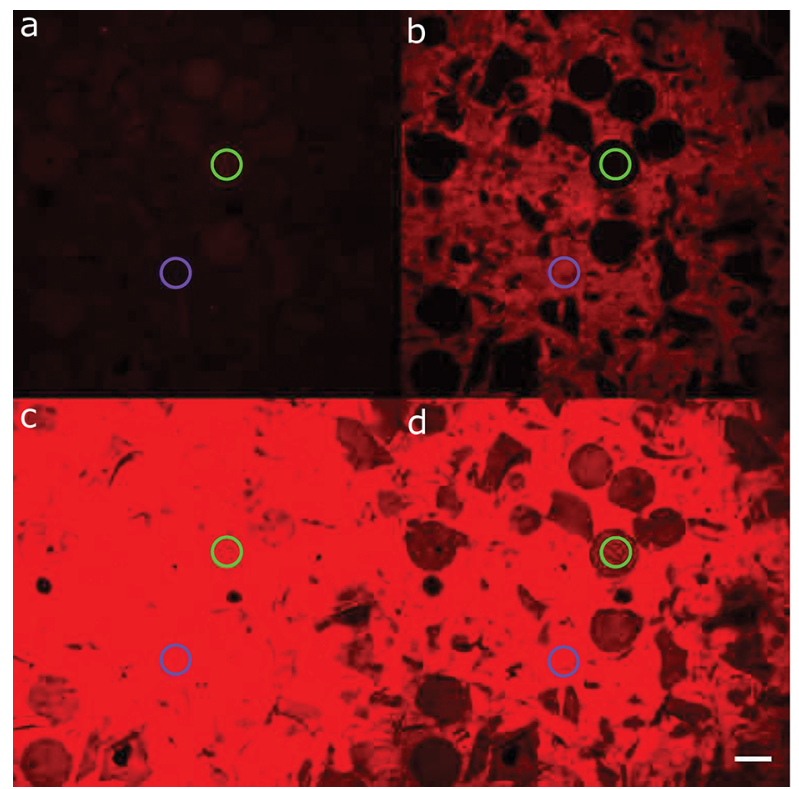

e

Figure 3. PAA containing composite imaged at wavenumbers ranging from 3400 to $2800 \mathrm{~cm}^{-1}$. Selected images at wavenumbers $3184,2997,2905$, and $2830 \mathrm{~cm}^{-1}$ are presented in (a) $-(\mathrm{d})$, respectively. The spectra (e) illustrate the signal from the PAA particles and the matrix.

3.1. Impedance Spectroscopy of PAA Containing Composites. PAA containing composites, with thicknesses of $550 \pm 25 \mu \mathrm{m}$ and $1050 \pm 50 \mu \mathrm{m}$, were investigated by using impedance spectroscopy, while exposed to a $154 \mathrm{mM}$ sodium chloride solution. The results are displayed in Figure 2 as Bode plots (Figure 2a,b) and impedance-time plots at fixed frequency, $f=100 \mathrm{kHz}$ (Figure $2 \mathrm{c}, \mathrm{d}$ ). At short times (20 min) after the sample was exposed to salt solution, the Bode plots show that the impedance is inversely proportional to the frequency, which indicates purely capacitive behavior. For capacitive behavior, $|Z|=\frac{1}{i \omega C}$, the proportionality constant theoretically is -1 in a $\log -\log$ plot of impedance vs frequency. ${ }^{32}$ The slope of the linear frequency sweeps was determined to be $-0.954 \pm 0.010$, which is in agreement with the theoretical value of -1 . The reported value represents an average and standard deviation of five independent frequency sweeps. The capacitive behavior is also observed in the impedance-time plots where the phase angle, $-\phi$, is close to $90^{\circ} .{ }^{33}$ Over time, the saline solution penetrates into the composites through the hydrophilic particles, resulting in a steady decrease in impedance. After some time, sudden drops are observed in both the impedance and the phase angle for all composites. This means the composites no longer behave purely capacitive and implies that saline solution has fully penetrated the composites, i.e., reached the working electrode. The phase angle is less sensitive to small variations in the capacitance compared to the magnitude of the impedance. The time of full penetration, $t_{\mathrm{p}}$, is therefore arbitrarily but consistently defined as the time at which the phase angle has decreased by $5 \%$ from its original value, $\phi_{\mathrm{p}}=0.95 \phi_{0}$. After full penetration of the saline solution corrosion may occur at the surface the working electrode. This increases the complexity of the obtained data, and we will therefore limit our diffusion analysis to times much shorter than the penetration time. The impedance and phase angle continue to change after full penetration in all cases. This indicates the composites are not saturated at that point and continue to absorb saline solution. The impedance eventually reaches constant values, $|Z| \sim 20 \Omega$, which is comparable to the impedance of the electrolyte solution. This means the composites have absorbed enough saline solution to exhibit impedance values similar to the free saline solution.

Quantitative information about the diffusion kinetics can be extracted from impedance data by making appropriate assumptions about the sample capacitance. One approach represented in the literature is the Brasher-Kingsbury model where the capacitive changes are attributed to changes in the system's dielectric properties, while the dielectric thickness is assumed to remain constant. ${ }^{34}$ The water uptake can subsequently be estimated by calculating the dielectric properties relative to the dielectric constant of water. The Brasher-Kingsbury model assumptions work well for systems absorbing low quantities of water, typically $<10 \mathrm{vol} \%$, and have been successfully applied to evaluate water diffusion kinetics in protective coatings. ${ }^{35-38}$ Because the impedance values of our systems reach values comparable to the electrolyte solution for long immersion times, the water content is expected to be high why the Brasher-Kingsbury model is not expected to be suitable. However, to find the most appropriate model conditions for water diffusion in our materials, complementary techniques are needed. Here, we use CARS microscopy to evaluate the composite during water exposure to identify appropriate modeling assumptions.

3.2. Imaging Water Diffusion Using CARS Microscopy. PAA containing composites were imaged by using CARS microscopy. To identify energies resulting in high particle-matrix contrast, composites were imaged at wavenumbers, $\tilde{\nu}$, ranging from 3400 to $2800 \mathrm{~cm}^{-1}$, which cover the $\mathrm{O}-\mathrm{H}$ and some of the $\mathrm{C}-\mathrm{H}$ stretching regions. Figures $3 \mathrm{a}-\mathrm{d}$ show resulting images collected at different wavenumbers indicated by letters $(a-d)$ in the spectra (Figure $3 e)$. The dashed lines in the spectra indicate the intensity difference between PAA and the matrix. Similar intensity differences were obtained at several different wavenumbers; however, the highest relative contrast between PAA and matrix was found at $\tilde{\nu}=2997 \mathrm{~cm}^{-1}$. Here, the aromatic groups of the styrene in SIS give a high intensity signal from the matrix, while the PAA 
particles do not exhibit molecular vibrations corresponding to this energy due to the lack of aromatic double bonds and thus remain dark in the images.

Image sequences were acquired at $\tilde{\nu}=2997 \mathrm{~cm}^{-1}$ during water exposure (Figure 4). The water was supplied as

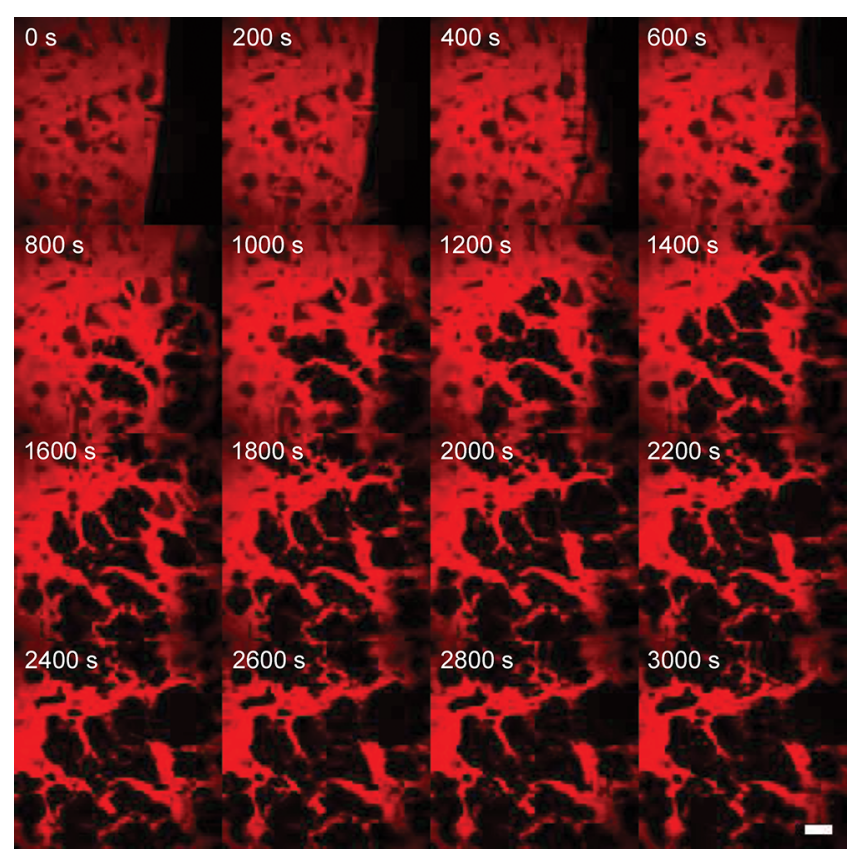

Figure 4. CARS image montage of a sample exposed to water. The composite in the montage contains 40 wt \% PAA particles, which appear to swell as water propagates in the sample. The scale bar is 100 $\mu \mathrm{m}$.

illustrated in Figure 1b. Here, the evolution of the water transport is observed as swelling of the dark regions corresponding to the hydrophilic PAA particles, which shows that the particles indeed are responsible for the water transport. The matrix (red region) is displaced due to particle swelling as the water front is propagating through the sample. Water does not contribute significantly to the signal at the measured wavenumber. To help visualize the particle swelling, the images were divided into segments perpendicular to the flow front direction. The segment width is $100 \mu \mathrm{m}$ (Figure 5), which is comparable to the particle size. The particle area of each segment was subsequently determined relative to the total segment area. The changes in relative particle area in each image segment were plotted as a function of time and are displayed in Figure 5. The plots show that the relative particle area in each segment is approximately $25-35 \%$ in the dry state, which is in agreement with the particle fraction of the mix at 27.1 vol \%. The relative particle area at saturation is around $90 \%$, implying each segment contains at least $60 \%$ water at saturation. This is in agreement with the low impedance at saturation, indicating that saturated sample segments are electrically conducting. The time between the start of swelling and saturation, indicated by arrows in Figure 5, is $18 \mathrm{~min}$ for the first segment $(0-100 \mu \mathrm{m})$ and $30 \mathrm{~min}$ for the third segment $(200-300 \mu \mathrm{m})$. This indicates that the concentration profile of the flow front broadens over time. The initial swelling time of the third segment happens after $\sim 18-20 \mathrm{~min}$, which seems to agree with the impedance measurements where full penetration of a $565 \mu \mathrm{m}$ PAA containing composite happens after $28.9 \mathrm{~min}$. In the following section this information will be used to model the impedance data for two different particle containing composites.

3.3. Diffusion Modeling and Material Variation. From the CARS imaging we found that PAA containing composites exposed to water consist of a dry part, a semiwetted part, and a saturated part. Furthermore, the fraction of each part changes as a function of time. The impedance data and CARS images indicate that saturated sample segments can be assumed to be perfectly conducting, which results in an overall decrease of the effective dielectric thickness. The Brasher-Kingsbury model is not found suitable for data interpretation of these composites since it assumes constant dielectric thickness. Instead, we define an effective water penetration front that includes contributions from saturated, semiwetted, and dry sample segments. The effective water penetration front is here defined as $\hat{l}=1-\frac{C_{0}}{C_{t}}$, where $C_{0}$ is the initial capacitance and $C_{t}$ is the capacitance at time, $t$. From the definition of parallel plate capacitance, $C=\frac{\varepsilon_{0} \varepsilon_{\mathrm{r}} A}{l}$, we obtain

$$
\hat{\imath}=1-\frac{\varepsilon_{\mathrm{r}, 0}}{l_{0}} \frac{l_{t}}{\varepsilon_{\mathrm{r}, t}}
$$

Here, $\varepsilon_{\mathrm{r}, 0}$ and $\varepsilon_{\mathrm{r}, t}$ are dielectric constants and $l_{0}$ and $l_{t}$ are thicknesses of the sample at time $t=0$ and $t$, respectively. This model is valid for times shorter than the time of full penetration, $t<t_{\mathrm{p}}$, after which the electrical properties of the substrate are expected to have a significant contribution. The diffusion kinetics are therefore extracted for times much shorter than the time of full penetration, $t \ll t_{\mathrm{p}}$. The simplest description of capacitive changes for a highly water absorbing system is to consider a binary water concentration profile, where a given sample segment is either dry or fully saturated. The dielectric constant of the sample can then effectively be considered constant $\varepsilon_{\mathrm{r}, t}=\varepsilon_{\mathrm{r}, 0}$, while only the dielectric thickness changes over time. Equation 2 is readily reduced to $\hat{l}=\frac{l_{0}-l_{t}}{l_{0}}$ describing the temporal water penetration. However, CARS observations showed that the water concentration profile is a gradient rather than binary. The dielectric constant must therefore vary spatially with the water concentration, $\varepsilon_{\mathrm{r}, t}$ $=\varepsilon_{\mathrm{r}, t}(x)$ (1D diffusion). The effective water penetration front (eq 2) was determined from the temporal capacitance at 100 $\mathrm{kHz}$ for increased sensitivity to changes in dielectric properties. The temporal capacitance was calculated via eq 1 . The effective water penetration front for PAA containing composites is plotted as a function of time in Figure 6. The curves hold information about the water diffusion kinetics, which can be extracted by fitting a power law (eq 3) previously found useful to classify the water diffusion mechanism in polymeric systems. $^{21,39}$

$$
\hat{\imath}=k t^{n}
$$

where $k$ is a proportionality constant related to the diffusion rate and sample geometry and $n$ is the diffusional exponent indicating the diffusion mechanism. The diffusion model is fitted to data up to half of the full penetration of the thinnest sample for each sample type since the diffusion mechanism ideally is independent of sample thickness. ${ }^{40}$ This also ensures that there is no capacitive contributions from the working electrode, since there is a dry polymer layer with a thickness of at least $\sim 250 \mu \mathrm{m}$ covering the stainless steel electrode. The 


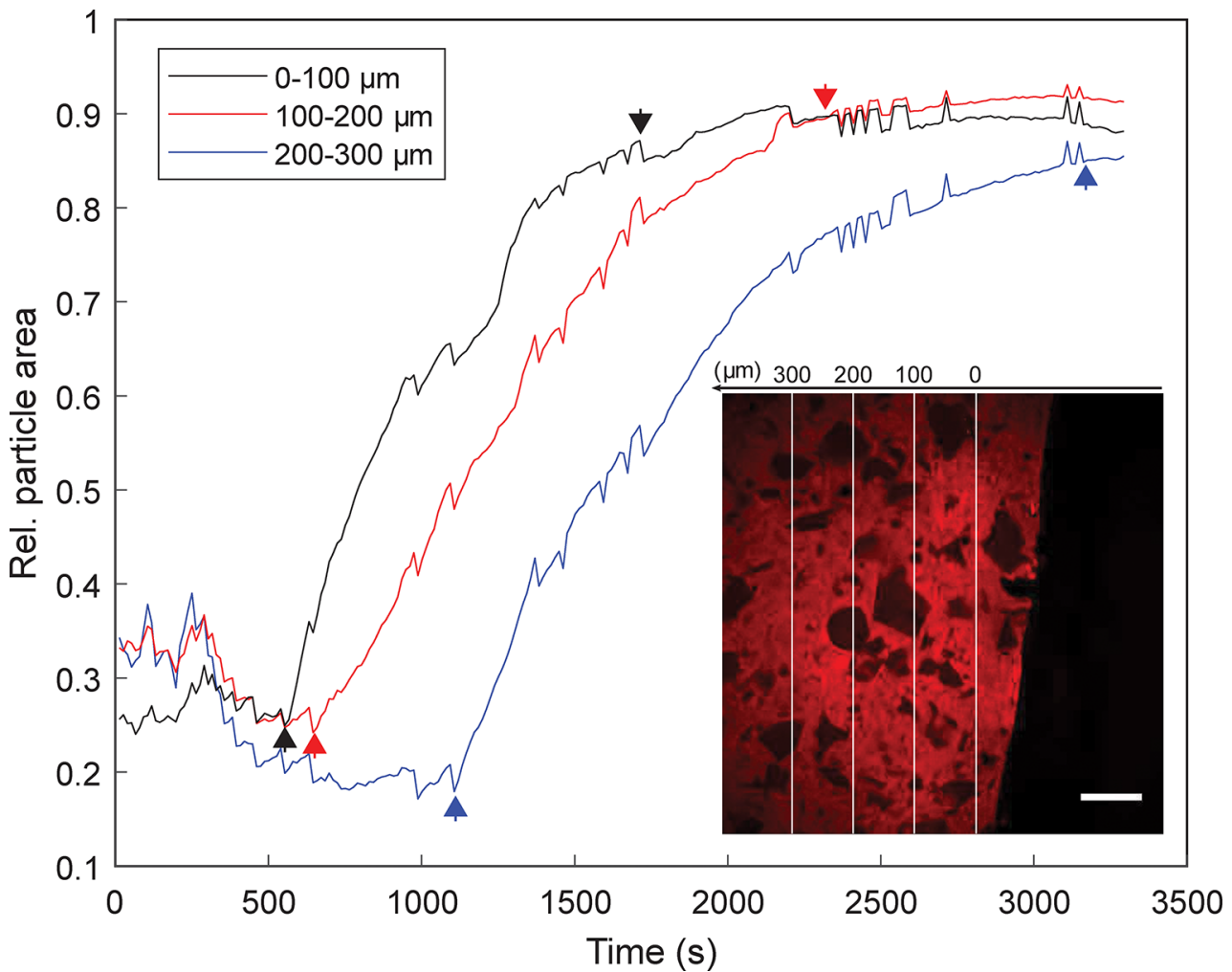

Figure 5. Particle area relative to the total segment area plotted as a function of time for each segment. The vertical white lines illustrate the boundaries of the segments having widths of $100 \mu \mathrm{m}$. The arrows indicate swelling initiation and saturation of each segment. The scale bar is 100 $\mu \mathrm{m}$.
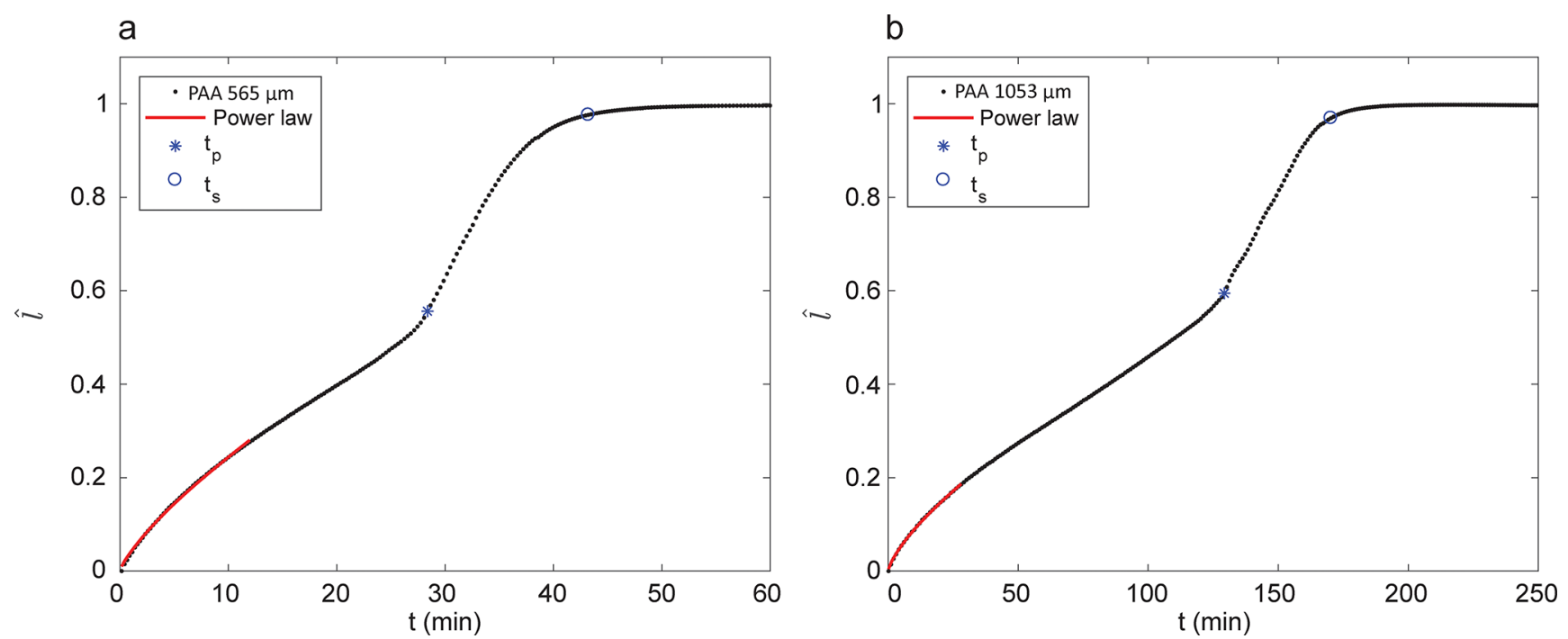

Figure 6. Effective water penetration front plotted as a function of time for each sample type: $565 \mu \mathrm{m}$ PAA (a) and $1053 \mu \mathrm{m}$ PAA (b). The fits represent a power law fitted to the data up to half-penetration of the thinnest sample. The points of full penetration $(*)$ and saturation $(O)$ are also indicated.

point of full penetration $(*)$ and the point of saturation $(O)$ are marked in Figure 6. Saturation is arbitrarily but consistently defined as the point where the effective water penetration front is $2 \%$ from its maximum value. The time of saturation is denoted $t_{s}$. The difference between the saturation and full penetration time, $\Delta t=t_{\mathrm{s}}-t_{\mathrm{p}}$, reflects the width of the concentration profile. $\Delta t$ increases with increasing thickness, from $\Delta t(565 \mu \mathrm{m})=16.6 \pm 1.6 \mathrm{~min}$ to $\Delta t(1053 \mu \mathrm{m})=35.9 \pm$ $3.6 \mathrm{~min}$. This implies that the width of the concentration profile is increasing over time, which is in agreement with the CARS observations.

Next, CHEC containing composites with the same PIB-SIS matrix and particle loading were examined. The impedance data, provided as Supporting Information (Figure S2), reached low values similar to the PAA containing composites. This means that these composites also absorb amounts of water, which makes the composites electrically conducting. The data are therefore analyzed following the same approach as for the 


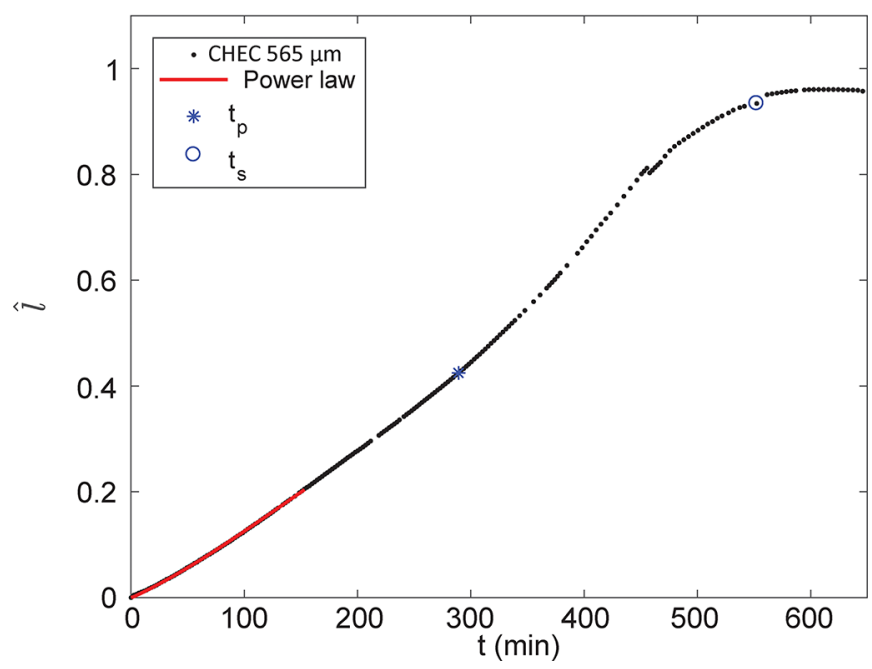

b

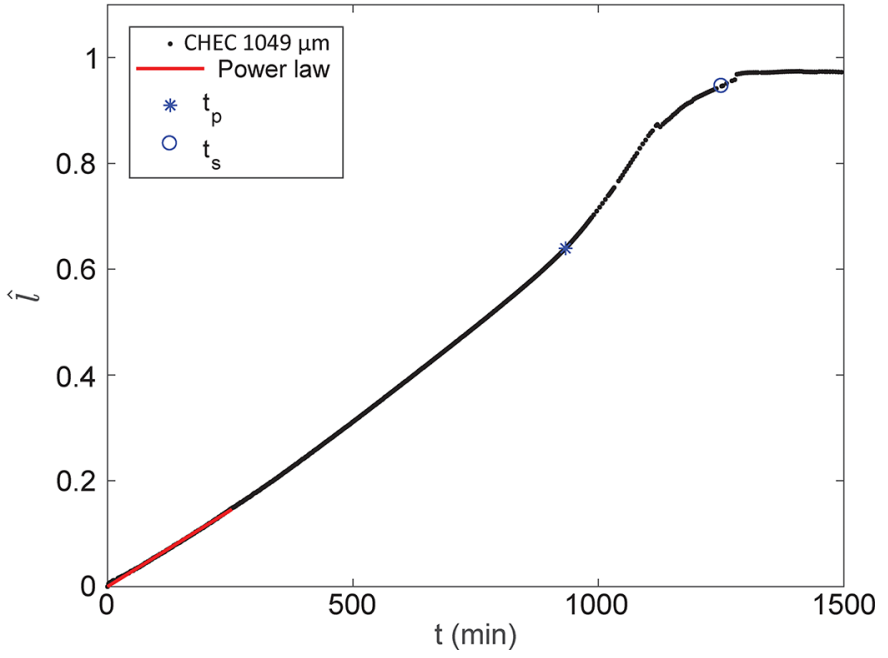

Figure 7. Effective water penetration front plotted for each sample type: $565 \mu \mathrm{m}$ CHEC (a) and $1049 \mu \mathrm{m}$ CHEC (b). The fits represent a power law fitted to the data up to half-penetration of the thinnest sample. The points of full penetration and saturation are marked $(*)$ and $(O)$, respectively.

Table 1. Penetration Times, Saturation Times, Diffusional Exponents, and Proportionality Constants Summarized for the Four Different Composites ${ }^{a}$

\begin{tabular}{lcccrr} 
type & $l_{0}(\mu \mathrm{m})$ & $t_{\mathrm{p}}(\mathrm{min})$ & $t_{\mathrm{s}}(\mathrm{min})$ & \multicolumn{1}{c}{$\Delta t(\mathrm{~min})$} & $k\left(\mathrm{~s}^{-n}\right)$ \\
PAA & 565 & $28.9 \pm 1.0$ & $45.6 \pm 2.6$ & $16.6 \pm 1.6$ & $0.77 \pm 0.01$ \\
PAA & 1053 & $141.0 \pm 10.3$ & $176.9 \pm 8.0$ & $35.9 \pm 3.6$ & $0.68 \pm 0.03$ \\
CHEC & 565 & $253 \pm 28$ & $507 \pm 36$ & $254 \pm 28$ & 0.08 \\
CHEC & 1049 & $766 \pm 119$ & $1151 \pm 72$ & $385.0 \pm 48$ & $0.04 \pm 0.11$
\end{tabular}

${ }^{a}$ Each value represents an average of three results with their respective standard deviation.

PAA containing composites. The effective water penetration front for the CHEC containing composites is plotted as a function of time in Figure 7 for composites with thicknesses of $565 \mu \mathrm{m}$ (Figure 7a) and $1049 \mu \mathrm{m}$ (Figure 7b). All impedancederived results are summarized in Table 1 where the reported values are averages of triplicates with their respective standard deviations. The CHEC containing composites also indicate flow front broadening since $\Delta t$ increases with increasing sample thickness, i.e., from $\Delta t(565 \mu \mathrm{m})=254.0 \pm 28.0 \mathrm{~min}$ to $\Delta t(1053 \mu \mathrm{m})=385.0 \pm 47.5 \mathrm{~min}$. The CHEC containing composites have longer penetration and saturation times compared to the PAA containing composites. This means the CHEC particles transport water less effectively than the PAA particles assuming similar particle dispersion. This implies that the water affinity of the PAA particles is higher compared to the CHEC particles, which is in agreement with the chemical structure of PAA and CHEC. Diffusional exponents of the PAA containing composites are $n=0.77 \pm 0.001$ and $n=0.68$ \pm 0.03 for the 565 and $1049 \mu \mathrm{m}$ composites, respectively. The CHEC containing composites have diffusional exponents of $n$ $=1.04 \pm 0.11$ and $n=0.97 \pm 0.03$ for the 565 and $1049 \mu \mathrm{m}$ composites, respectively. The diffusional exponents should ideally be independent of sample thickness; however, minor variations are observed for the PAA containing composites. We speculate that it is due to the thickness of the thin composites approaching the particle size, potentially leading to increased influence of variations in filler dispersion along the composite thickness. The largest variations in diffusional exponents are, however, found between the PAA and the CHEC containing composites. The PAA containing composites exhibit anom- alous diffusion $(0.5<n<1)$, while the CHEC containing composites demonstrate case II sorption $(n \approx 1)$. The anomalous mechanism is characteristic for systems where both Fickian and relaxation-restricted diffusion processes occur simultaneously. Similar diffusional exponents, obtained through gravimetric measurements, have previously been reported for PAA in the literature. ${ }^{41}$ Case II sorption is characteristic for systems where the penetrant diffusion is highly restricted by polymer relaxation processes. ${ }^{39}$ This implies the polymer relaxation processes are slower for CHEC compared to PAA. The proportionality constant, $k$, is only of physical significance for limiting cases, i.e., Fickian diffusion $(n=0.5)$ and case II sorption $(n=1) .^{42,43}$ For case II sorption the proportionality constant holds information about the polymer relaxation and the equilibrium water concentration, and it is inversely proportional to the thickness. Since we do not have knowledge about the equilibrium water concentration, meaningful information cannot be extracted from the proportionality constant. However, one can still compare the constants for each thickness of the CHEC containing composites to evaluate their consistency. This is readily done by multiplying the proportionality constants with their respective thicknesses: $k \cdot l_{0}(565 \mu \mathrm{m})=4.7 \times 10^{-5} \mathrm{~m} \mathrm{~s}^{-1}$ and $k \cdot l_{0}(1049 \mu \mathrm{m})=4.3 \times 10^{-5} \mathrm{~m} \mathrm{~s}^{-1}$. The two proportionality constants for the CHEC containing composites are in good agreement, which demonstrates that the impedance measurements in combination with the developed model is a consistent tool for probing water diffusion in the polymer composites. 


\section{CONCLUSION}

Impedance spectroscopy was used to evaluate water diffusion in PAA and CHEC containing polymer composites. To aid data interpretation, the diffusion process was imaged for selected PAA containing composites. This was done by monitoring the structural changes of the material during water absorption using CARS imaging. The CARS images showed that the water concentration in the composite was a gradient with the two extremes being dry polymer and highly water saturated polymer containing $60-90 \%$ water. An effective water penetration front was subsequently defined as the relative change in the temporal capacitance. Diffusion kinetics were successfully extracted from the effective water penetration front by fitting a semiempirical power law to the results. The diffusion was in all cases limited by polymer relaxation processes. However, the PAA containing composites showed anomalous diffusion, while the CHEC containing composites exhibited case II sorption. This means that the relaxation processes were faster for the PAA containing composites compared to the CHEC containing composites. Characteristic penetration and saturation times were determined and revealed that the concentration profile of the flow front broadens over time. Finally, aqueous solution was found to penetrate and saturate the PAA containing composites approximately an order of magnitude faster compared to the CHEC containing composites. This implies that PAA has higher water affinity than CHEC, as expected from water uptake data for the pure components. Apart from these simple model composites, the developed method can readily be used for examining more complex material formulations such as layered materials or composites containing multiple different filler types and plasticizers.

\section{ASSOCIATED CONTENT}

\section{S Supporting Information}

The Supporting Information is available free of charge at https://pubs.acs.org/doi/10.1021/acsapm.9b01107.

Impedance spectroscopy data for the CHEC containing composites (PDF)

\section{AUTHOR INFORMATION}

\section{Corresponding Author}

*E-mail: esth@kemi.dtu.dk.

\section{ORCID $\odot$}

Daniel Hansen: 0000-0001-6834-9450

Jonathan R. Brewer: 0000-0002-3444-1715

Esben Thormann: 0000-0002-2364-3493

\section{Notes}

The authors declare no competing financial interest.

\section{ACKNOWLEDGMENTS}

The Danish Molecular Biomedical Imaging Center (DaMBIC, University of Southern Denmark) is acknowledged for the use of the CARS microscope. The authors gratefully acknowledge financial support from Coloplast A/S and the Innovation Fund Denmark through the Grand Solutions Project \#6151-00007B. Niels Jerichau Clausen, Tanguy Floch, and Aamir Shabbir are acknowledged for helpful discussions regarding impedance spectroscopy.

\section{REFERENCES}

(1) Wang, J.; Xu, H.; Battocchi, D.; Bierwagen, G. The determination of critical pigment volume concentration (CPVC) in organic coatings with fluorescence microscopy. Prog. Org. Coat. 2014, $77,2147-2154$.

(2) Sumita, M.; Sakata, K.; Asai, S.; Miyasaka, K.; Nakagawa, H. Dispersion of fillers and the electrical conductivity of polymer blends filled with carbon black. Polym. Bull. 1991, 25, 265-271.

(3) Ferrari, F.; Bertoni, M.; Bonferoni, M. C.; Rossi, S.; Caramella, C.; Waring, M. J. Comparative evaluation of hydrocolloid dressings by means of water uptake and swelling force measurements: II. Int. J. Pharm. 1995, 117, 49-55.

(4) Hansen, D.; Bomholt, N.; Jeppesen, J. C.; Simonsen, A. C. Contact angle goniometry on single micron-scale fibers for composites. Appl. Surf. Sci. 2017, 392, 181-188.

(5) Jafarzadeh, S.; Claesson, P. M.; Sundell, P. E.; Pan, J.; Thormann, E. Nanoscale electrical and mechanical characteristics of conductive polyaniline network in polymer composite films. ACS Appl. Mater. Interfaces 2014, 6, 19168-19175.

(6) Jesus, C. R.; Molina, E. F.; Pulcinelli, S. H.; Santilli, C. V. Highly Controlled Diffusion Drug Release from Ureasil-Poly(ethylene oxide)-Na+-Montmorillonite Hybrid Hydrogel Nanocomposites. ACS Appl. Mater. Interfaces 2018, 10, 19059-19068.

(7) Fan, X. Mechanics of moisture for polymers: Fundamental concepts and model study. EuroSimE 2008 - International Conference on Thermal, Mechanical and Multi-Physics Simulation and Experiments in Microelectronics and Micro-Systems, 2008; pp 1-14.

(8) Kenney, J. F.; Haddock, T. H.; Sun, R. L.; Parreira, H. C. Medical-grade acrylic adhesives for skin contact. J. Appl. Polym. Sci. 1992, 45, 355-361.

(9) Pejcic, B.; De Marco, R. Impedance spectroscopy: Over 35 years of electrochemical sensor optimization. Electrochim. Acta 2006, 51, 6217-6229.

(10) Bajpai, A. K.; Giri, A. Water sorption behaviour of highly swelling (carboxy methylcellulose-g-polyacrylamide) hydrogels and release of potassium nitrate as agro-chemical. Carbohydr. Polym. 2003, 53, 271-279.

(11) Reuvers, N. J. W.; Huinink, H. P.; Adan, O. C. G.; Garcia, S. J.; Mol, J. M. C. Water uptake in thin nylon 6 films as measured by electrochemical impedance spectroscopy and magnetic resonance imaging. Electrochim. Acta 2013, 94, 219-228.

(12) Vlasak, R.; Klueppel, I.; Grundmeier, G. Combined EIS and FTIR-ATR study of water uptake and diffusion in polymer films on semiconducting electrodes. Electrochim. Acta 2007, 52, 8075-8080.

(13) Vosgien Lacombre, C.; Bouvet, G.; Trinh, D.; Mallarino, S.; Touzain, S. Water uptake in free films and coatings using the Brasher and Kingsbury equation: a possible explanation of the different values obtained by electrochemical Impedance spectroscopy and gravimetry. Electrochim. Acta 2017, 231, 162-170.

(14) Tian, W.; Meng, F.; Liu, L.; Li, Y.; Wang, F. The failure behaviour of a commercial highly pigmented epoxy coating under marine alternating hydrostatic pressure. Prog. Org. Coat. 2015, 82, 101-112.

(15) Fredj, N.; Cohendoz, S.; Feaugas, X.; Touzain, S. Some consequences of saline solution immersion on mechanical behavior of two marine epoxy-based coatings. Prog. Org. Coat. 2010, 69, 82-91.

(16) Fredj, N.; Cohendoz, S.; Mallarino, S.; Feaugas, X.; Touzain, S. Evidencing antagonist effects of water uptake and leaching processes in marine organic coatings by gravimetry and EIS. Prog. Org. Coat. 2010, 67, 287-295.

(17) Grammatikos, S. A.; Ball, R. J.; Evernden, M.; Jones, R. G. Impedance spectroscopy as a tool for moisture uptake monitoring in construction composites during service. Composites, Part A 2018, 105, $108-117$.

(18) Crank, J. A theoretical investigation of the influence of molecular relaxation and internal stress on diffusion in polymers. $J$. Polym. Sci. 1953, 11, 151-168.

(19) Van Der Wel, G. K.; Adan, O. C. Moisture in organic coatings a review. Prog. Org. Coat. 1999, 37, 1-14. 
(20) Crank, J. The Mathematics of Diffusion; Oxford University Press: 1975

(21) De Kee, D.; Liu, Q.; Hinestroza, J. Viscoelastic (Non-Fickian) Diffusion. Can. J. Chem. Eng. 2005, 83, 913-929.

(22) Thomas, N. L.; Windle, A. H. A theory of case II diffusion. Polymer 1982, 23, 529-542.

(23) Rehage, G.; Ernst, O.; Fuhrmann, J. Fickian and non-Fickian diffusion in high polymer systems. Discuss. Faraday Soc. 1970, 49, 208.

(24) Smith, P. M.; Fisher, M. M. Non-Fickian diffusion of water in melamine-formaldehyde resins. Polymer 1984, 25, 84-90.

(25) Esposito, F.; Del Nobile, M. A.; Mensitieri, G.; Nicolais, L. Water sorption in cellulosebased hydrogels. J. Appl. Polym. Sci. 1996, 60, 2403-2408.

(26) Sangermano, M.; Malucelli, G.; Bongiovanni, R.; Priola, A.; Annby, U.; Rehnberg, N. Influence of the $\mathrm{COOH}$ and $\mathrm{COONa}$ groups and crosslink density of poly(acrylic acid)/montmorillonite superabsorbent composite on water absorbency. Polym. Int. 2001, 50, $1050-1053$.

(27) Evans, C. L.; Xie, X. S. Coherent anti-Stokes Raman scattering microscopy: chemical imaging for biology and medicine. Annu. Rev. Anal. Chem. 2008, 1, 883-909.

(28) Drutis, D. M.; Hancewicz, T. M.; Pashkovski, E.; Feng, L.; Mihalov, D.; Holtom, G.; Ananthapadmanabhan, K. P.; Xie, X. S.; Misra, M. Three-dimensional chemical imaging of skin using stimulated Raman scattering microscopy. J. Biomed. Opt. 2014, 19, 111604.

(29) Glover, Z. J.; Ersch, C.; Andersen, U.; Holmes, M. J.; Povey, M. J.; Brewer, J. R.; Simonsen, A. C. Super-resolution microscopy and empirically validated autocorrelation image analysis discriminates microstructures of dairy derived gels. Food Hydrocolloids 2019, 90, $62-71$.

(30) Zumbusch, A.; Holtom, G. R.; Xie, X. S. Three-dimensional vibrational imaging by coherent anti-Stokes Raman scattering. Phys. Rev. Lett. 1999, 82, 4142.

(31) Ibata, K.; Takimoto, S.; Morisaku, T.; Miyawaki, A.; Yasui, M. Analysis of aquaporin-mediated diffusional water permeability by coherent anti-stokes Raman scattering microscopy. Biophys. J. 2011, 101, 2277-2283.

(32) Allahar, K. N.; Hinderliter, B. R.; Tallman, D. E.; Bierwagen, G. P. Water Transport in Multilayer Organic Coatings. J. Electrochem. Soc. 2008, 155, F201.

(33) Amirudin, A.; Thieny, D. Application of electrochemical impedance spectroscopy to study the degradation of polymer-coated metals. Prog. Org. Coat. 1995, 26, 1-28.

(34) Brasher, D.; Kingsbury, A. Electrical Measurements in the Study of Immersed Paint Coatings on Metal. 1. Comparison between Capacitance and Gravimetric Methods of Estimating Water-Uptake. J. Appl. Chem. 1954, 4, 62-72.

(35) Yuan, X.; Yue, Z. F.; Chen, X.; Wen, S. F.; Li, L.; Feng, T. EIS study of effective capacitance and water uptake behaviors of siliconeepoxy hybrid coatings on mild steel. Prog. Org. Coat. 2015, 86, 41-48.

(36) Rezaei, F.; Sharif, F.; Sarabi, A. A.; Kasiriha, S. M.; Rahmanian, M.; Akbarinezhad, E. Evaluating water transport through high solid polyurethane coating using the EIS method. Journal of Coatings Technology Research 2010, 7, 209-217.

(37) van Westing, E. P.; Ferrari, G. M.; de Wit, J. H. The determination of coating performance with impedance measurementsI. Coating polymer properties. Corros. Sci. 1993, 34, 1511-1530.

(38) Castela, A.S.; Simöes, A.M. An impedance model for the estimation of water absorption in organic coatings. Part I: A linear dielectric mixture equation. Corros. Sci. 2003, 45, 1631-1646.

(39) Raucher, D.; Sefcik, M. D. Sorption and Transport in Glassy Polymers. Industrial Gas Separations 1983, 223, 111-124.

(40) Devanathan, M. A. V.; Stachurski, Z. The Adsorption and Diffusion of Electrolytic Hydrogen in Palladium. Proc. R. Soc. London, A 1962, 270, 90-102.

(41) Jabbari, E.; Nozari, S. Swelling behavior of acrylic acid hydrogels prepared by $\gamma$-radiation crosslinking of polyacrylic acid in aqueous solution. Eur. Polym. J. 2000, 36, 2685-2692.
(42) Peppas, N. A.; Brannon-Peppas, L. Water diffusion and sorption in amorphous macromolecular systems and foods. J. Food Eng. 1994, 22, 189-210.

(43) Ritger, P. L.; Peppas, N. A. A simple equation for description of solute release II. Fickian and anomalous release from swellable devices. J. Controlled Release 1987, 5, 37-42. 